\title{
Magnetic Loss Inside Solid and Laminated Components under Extreme Excitations
}

\author{
Zhiguang Cheng ${ }^{1}$, Behzad Forghani ${ }^{2}$, Yang Liu ${ }^{1,3}$, Yana Fan ${ }^{1}$, Tao Liu ${ }^{1}$, Zhigang Zhao ${ }^{4}$ \\ ${ }^{1}$ Institute of Power Transmission and Transformation Technology, Baobian Electric Co., Ltd, Baoding, China \\ ${ }^{2}$ Infolytica Corporation, Place du Parc, Montreal, Canada \\ ${ }^{3}$ State Grid Smart Grid Research Institute, Beijing, China \\ ${ }^{4}$ School of Electrical Engineering, Hebei University of Technology, Tianjin, China
}

Email address:

emlabzcheng@yahoo.com (Zhiguang Cheng), forghani@infolytica.com (B. Forghani), iamsam@hebut.edu.cn (Zhigang Zhao)

\section{To cite this article:}

Zhiguang Cheng, Behzad Forghani, Yang Liu, Yana Fan, Tao Liu, Zhigang Zhao. Magnetic Loss Inside Solid and Laminated Components Under Extreme Excitations. International Journal of Energy and Power Engineering. Special Issue: Numerical Analysis, Material Modeling and Validation for Magnetic Losses in Electromagnetic Devices. Vol. 5, No. 1-1, 2015, pp. 21-30. doi: 10.11648/j.ijepe.s.2016050101.13

\begin{abstract}
The modeling and numerical analysis of the magnetic loss inside components under multi-harmonic and/or DC-biasing excitations are increasingly of concern in large and special electromagnetic devices. This paper aims to investigate efficient and reliable approaches to determine the magnetic losses inside both the solid and laminated components under such extreme excitations. All the proposed approaches presented in the paper are experimentally validated.
\end{abstract}

Keywords: Benchmark Model, Components, Device-Based Model, Experimental Validation, Extreme Excitation, Finite Element Analysis(FEA), Magnetic Loss, Magnetic Material, Working Magnetic Properties

\section{Introduction}

Lately, there has been considerable focus on the modeling and prediction of the magnetic loss in electromagnetic devices, predominantly for the very high capacity and voltage level power equipment, operating under extreme excitations involving DC-biasing and/or multi-harmonic or PWM(Pulse Width Modulation) supplies [1-7].

However, the multi-scale nonlinear electromagnetic analysis and the related measurement of magnetic properties under such extreme excitations are quite challenging, additionally, making the validation of the modeling and numerical simulation very difficult, as compared to those under standard sinusoidal excitations or in the cases of lower capacity and voltage [8-9].

The purpose of this paper is to investigate efficient analysis and reliable experimental methods for evaluating the magnetic losses inside both the solid and laminated components in electromagnetic devices. The paper also addresses the working magnetic property modeling, and rigorous validation under extreme excitations, using the engineering-oriented test models and experimental setups with hybrid supply.

\section{Loss Determination under Extreme Excitation}

\subsection{Modeling and Computation of Magnetic Loss}

In the modeling and computation of the magnetic loss inside the solid and laminated components based on 3-D transient field solution under extreme excitations, including multi-harmonics and/or DC-AC hybrid supply, there are a number of key treatments and simplifications, including: 1) control of the identity of the applied excitation conditions in the experiment with that used in the transient field modeling; 2) measurement of the working magnetic property under applied excitation, at different DC-bias levels and/or with different harmonic contents; 3) non-uniformity of both the magnetic field and loss inside the solid plate due to the considerable skin effect and the laminated core-frame, caused by the lamination-joints; 4) accurate computation of the exciting coil's loss for the indirect determination of the magnetic loss in components; 5) comparison among different magnetization curves has shown that the DC magnetization curve can be practicably used in transient field analysis with hybrid excitation[10,11]; 6) for the laminated frame, if the induced 
eddy currents caused by the flux normal to the laminations are rather weak, then the additional magnetic loss in the frame can be neglected.

\subsection{Indirect Determination of Magnetic Loss}

In general, the magnetic loss $P_{\text {iron }}$ inside components cannot be measured directly, but, it can be determined indirectly through the measured total loss, referred to as on-load loss, $P_{\text {on-load }}$, and another loss component generated in the exciting coils $P_{E \text {-coil }}$. If the total loss has been measured, the problem is reduced to determining the loss in the air-core exciting coils specially designed for the purpose of the validation.

In this way, three approaches for determining $P_{E \text {-coil }}$ in the exciting coils, mainly dependent on the excitation level and the complexity of the proposed models, have been developed and realized by the authors.

1) Approach $I$

In the case of a weak single sinusoidal excitation and a low rated frequency, the leakage flux linked with the exciting coils does not change considerably with or without electromagnetic components. Thus the exciting coil's loss, without the electromagnetic components, can be directly measured, referred to as 'no-load' loss, $P_{E-\text { coil }}$, and then $P_{\text {iron }}$ in the magnetic components can be determined, as shown in (1),

$$
P_{\text {iron }}=P_{\text {on-load }}-\left.P_{E-\text { coil }}\right|_{\text {measured without electromag netic components }}
$$

\section{2) Approach II}

In the case of a strong excitation, the effect of the magnetic components on the leakage flux of the exciting coils must be considered. However, it is possible to use flux compensation to effectively determine $P_{E \text {-coil }}$. As an example, in a magnetic shield model, the exciting coils are located on one side of magnetic shield, which can be referred to as a high permeability plane with low eddy current reaction. In order to keep the leakage flux almost unaffected when the magnetic shield is removed, the compensatory coils, which have the completely same specifications as the exciting coils, are set up symmetrically on the other side of the high permeability plane [12].

The exciting coil's loss is measured using the leakage magnetic flux compensation coils, referred to as C-coils, and then $P_{\text {iron }}$ in the magnetic components is determined, as shown in (2),

$$
P_{\text {iron }}=P_{\text {on-load }}-\left.\left(\frac{P_{E-\text { coil }}+P_{C-\text { coil }}}{2}\right)\right|_{\text {measured with C-coil }}
$$

where $P_{E-\text { coil }}$ and $P_{\text {C-coil }}$ are induced in E-Coil and C-coil respectively.

\section{3) Approach III}

In the case of extreme excitation, including DC-biasing and/or multi-harmonic, and when the test model has a complicated magnetic structure, it is impossible to use Approaches I and II stated above. Fortunately, the exciting coil's loss $P_{E-\text {-coil }}$ can be accurately calculated based on a 3-D transient field solution under complicated excitation. Therefore the magnetic loss in magnetic components $P_{\text {iron }}$ can be determined by the following relation (3),

$$
P_{\text {iron }}=P_{\text {on-load }}-\left.\int_{\Omega_{c u}} \frac{\boldsymbol{J} \cdot \boldsymbol{J}}{\sigma} d v\right|_{\text {calculated based on 3-D transient field solution }}
$$

Note that the second term of (3) is for the calculation of the total loss of the exciting coil, $P_{E-\text {-coil }}$, including the eddy current loss and resistive loss caused in the exciting coil[13]. The relation (3) can now be used to indirectly determine the magnetic losses inside complex structures under extreme excitations.

\section{Magnetic Loss in Solid Components under Multi-Harmonic Excitation}

In this Section, the magnetic losses inside the solid components under multi-harmonics excitations are evaluated based on an upgraded benchmark model, $\mathrm{P} 21^{0}-\mathrm{B}^{+}[12,13]$.

\subsection{Magnetic Property Modeling of Magnetic Steel under Harmonic Excitation}

The specific total loss of the magnetic steel plate has been measured under multi-harmonic excitations by using ring specimens [14], and two specimens prepared for comparing magnetic properties between different ring sizes. The conductivity of the magnetic steel (Q235B), $\sigma=5.7895 \times 10^{6}$ $\mathrm{S} / \mathrm{m}$, and the dimensions of the two ring specimens (RS1 and RS2) are shown in Table 1.

Table 1. Dimensions of ring specimens.

\begin{tabular}{llll}
\hline Specimens & $\begin{array}{l}\text { Inner diameters } \\
(\mathbf{m m})\end{array}$ & $\begin{array}{l}\text { Outer Diameters } \\
(\mathbf{m m})\end{array}$ & $\begin{array}{l}\text { Thickness } \\
(\mathbf{m m})\end{array}$ \\
\hline RS1 & 330 & 400 & 10 \\
RS2 & 430 & 500 & 10 \\
\hline
\end{tabular}

The multi-harmonic voltage supply $U$ is defined in (4),

$$
U=U_{m 1} \sum_{k=1}^{4} c_{k} \sin [(2 k-1) \omega t]
$$

where $U_{m l}$ is the peak value of the fundamental voltage, and $c_{k}=1,0.3,0.5,0.4$ as $\mathrm{k}=1,2,3,4$, respectively. The waveforms of multi-harmonic supply are shown in Fig.1.

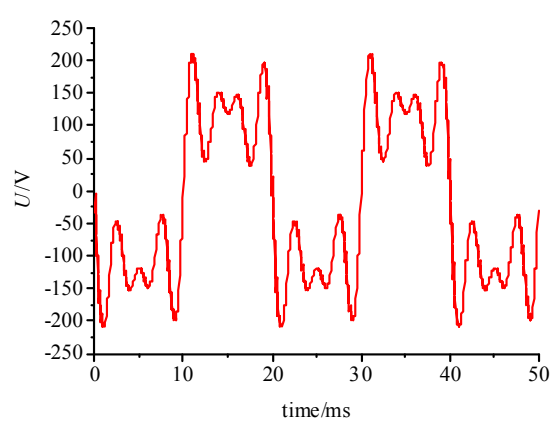

(a) 


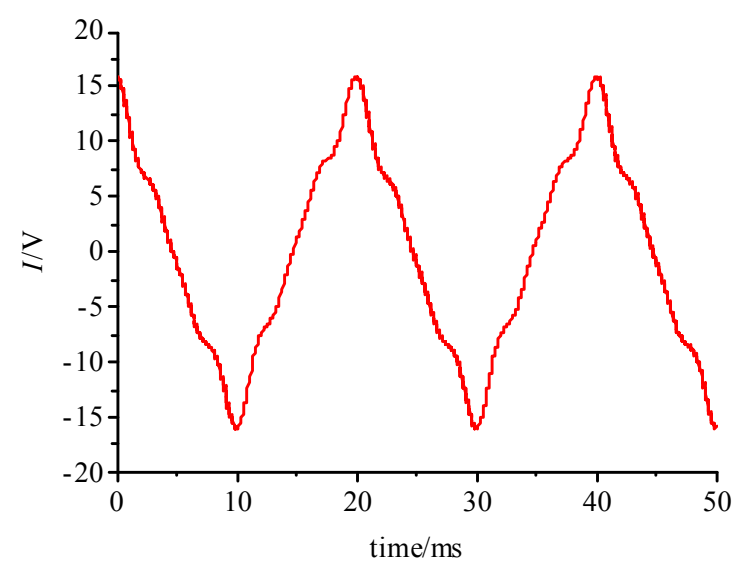

(b)

Figure 1. Multi-harmonic supply, (a) waveform of multi-harmonic voltage, (b) waveform of multi-harmonic current.

The block diagram of the circuit for measuring the magnetic properties, where the components include multifunction generator, WF1974, precision power amplifier, 4520/4520A, NF, power analyzer, WT3000, Yokogawa, and multi-channel temperature recorder, TP700, Toprie Electronic, are shown in Fig.2.

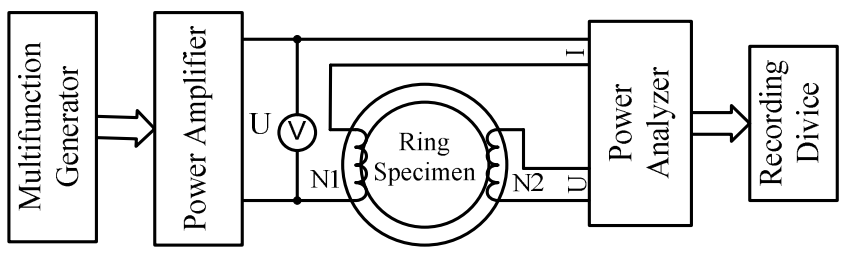

(a)

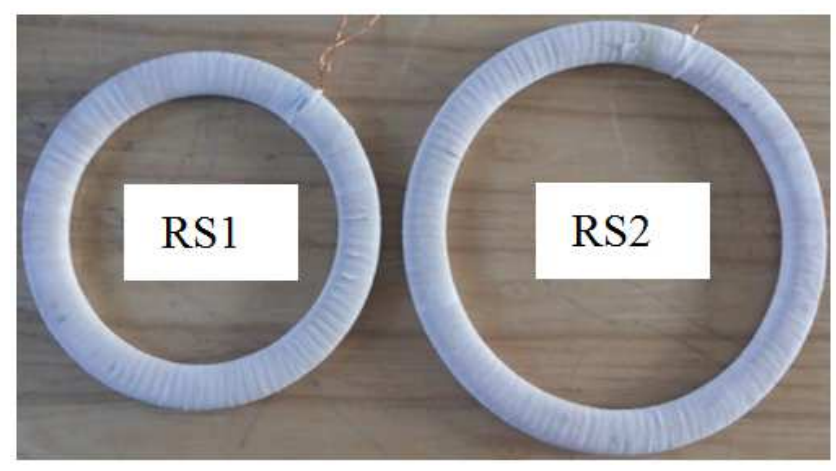

(b)

Figure 2. Measurement of magnetic properties using ring specimens under multi-harmonics (a) block diagram of the circuit; (b) ring specimens(RS1 \& $R S 2)$.

The measured average specific total loss curves, $W_{a v}-B_{m}$, with $3^{\text {rd }}, 5^{\text {th }}$ and $7^{\text {th }}$ harmonics, using two ring specimens, are shown in Fig.3. A minor difference can be seen at higher flux densities.

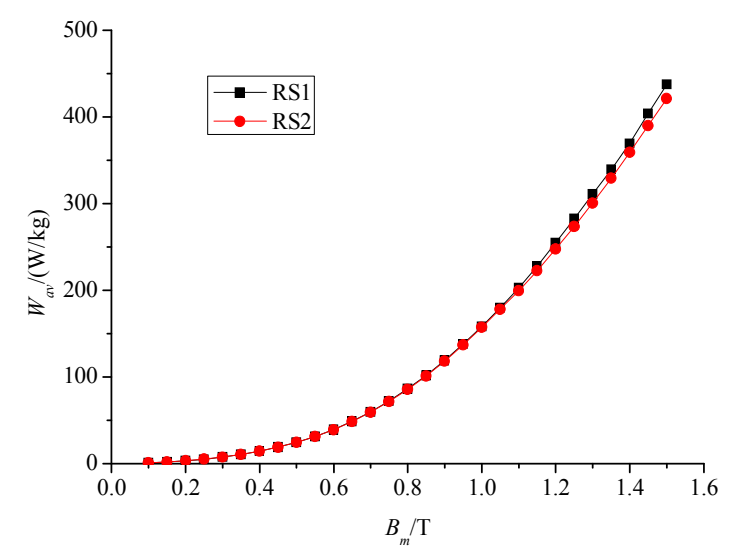

Figure 3. Average specific total loss curve under multi-harmonic excitation $(Q 235 B)$.

The DC magnetization curve (Q235B) is used in the transient field analysis, as shown in Fig.4, provided by the WISCO, Wuhan.

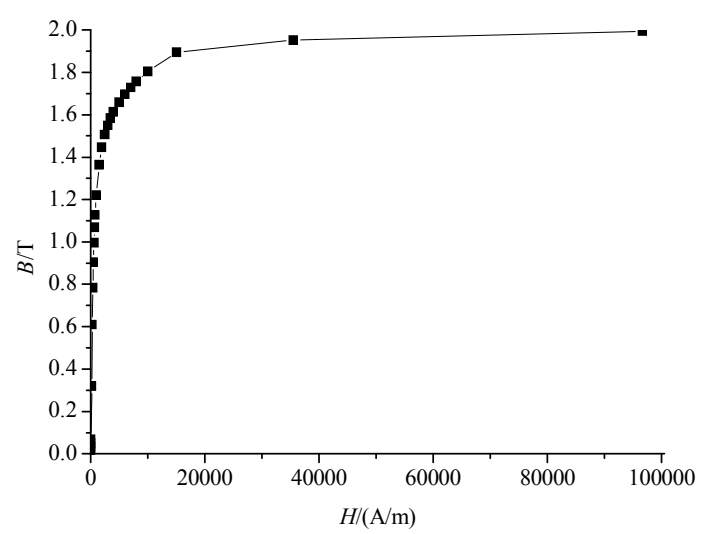

Figure 4. DC magnetization curve (Q235B).

\subsection{Upgraded TEAM Model and Loss Evaluation}

\subsubsection{Upgraded TEAM Benchmark Model}

Three decades ago, the TEAM benchmarking activities, now under the ICS (International Compumag Society) control, started to spread worldwide and has played a significant role in the progress of computational electromagnetic, especially for the validation of electromagnetic analysis methods. The engineering-oriented TEAM Problem 21 family, with a great deal of industrial involvement, is well established and has evolved significantly over time $[13,15,16]$. It is still being improved to deal with modeling and simulation under extreme excitations, making it very useful for both the development and the validation of more efficient analysis methods.

To model higher saturation levels, by increasing the excitation, or to use more complex excitation waveforms, the upgraded benchmark model $\mathrm{P} 21^{0}-\mathrm{B}^{+}$, based on the original member model P2 $1^{0}$-B of Problem 21 family, has two leakage flux generators (E-coils 1 and 2) and two leakage flux compensators (C-coils 1 and 2) [12,13], and the magnetic plate $(\mathrm{Q} 235 \mathrm{~B}$, size: $10 \times 500 \times 1000 \mathrm{~mm})$, as shown in Fig.5. 


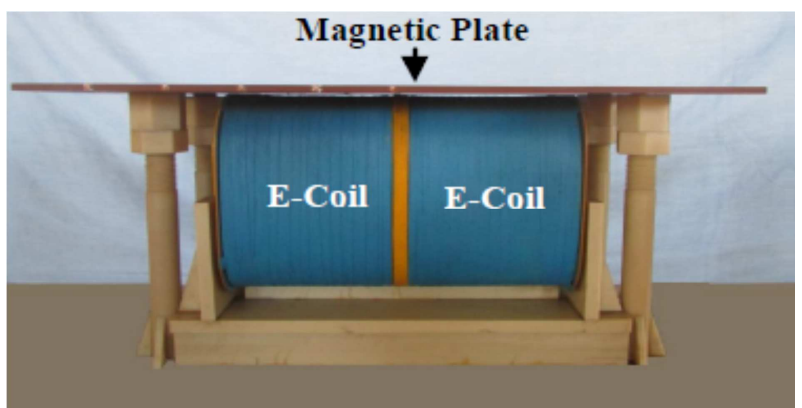

(a)

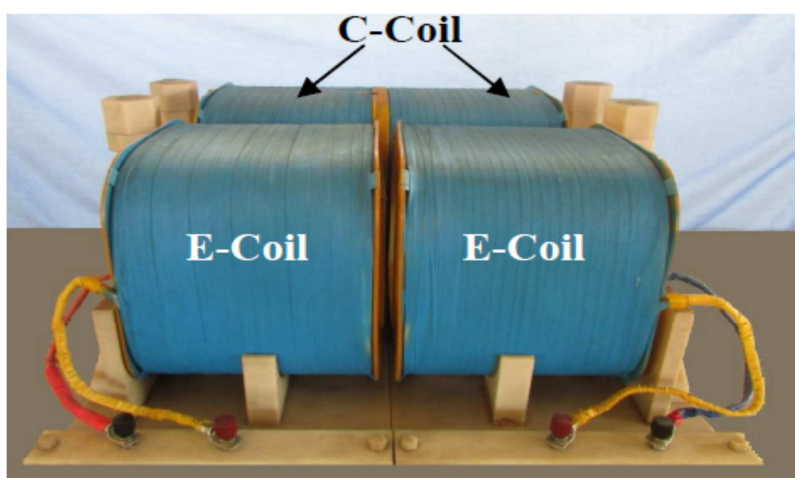

(b)

Figure 5. Loss measurement with upgraded E-coil and moveable C-coils $\left(P 2 I^{0}-B^{+}\right)$, (a) on-load case with magnetic plate, (b) no-load case with leakage flux compensation but without magnetic component.

\subsubsection{Loss Evaluation}

The total loss of the entire test model, $\mathrm{P} 21^{0}-\mathrm{B}^{+}$, is measured under multi-harmonic excitations; the measurement system is shown in Fig.6.

The loss caused in the air-core exciting coils of model $\mathrm{P} 21^{0}-\mathrm{B}^{+}$, without the magnetic plate, is calculated based on a 3-D transient field solution under either only sinusoidal or multi-harmonic excitations. The 3-D FE model of the exciting coil, using MagNet, Infolytica[13], is shown in Fig.7.

The magnetic loss inside the magnetic plate of $\mathrm{P} 21^{0}-\mathrm{B}^{+}$is calculated based on the 3-D transient field solution and the measured specific total loss data.

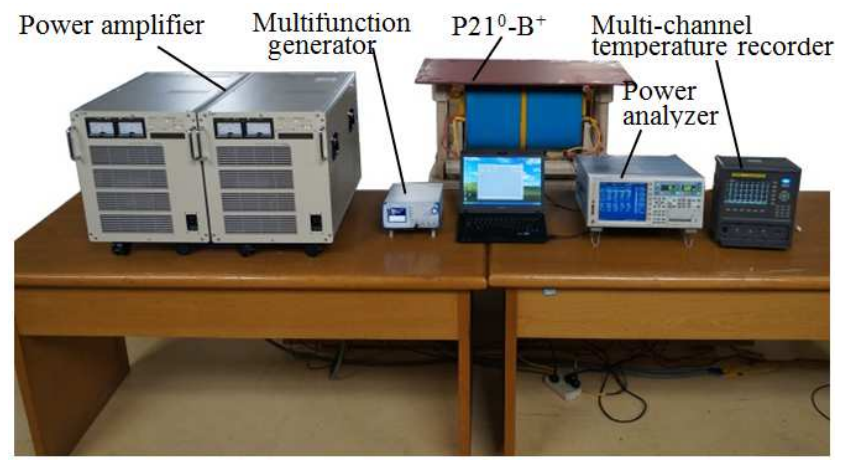

Figure 6. Measurement of magnetic loss in magnetic components under multi-harmonic excitation.
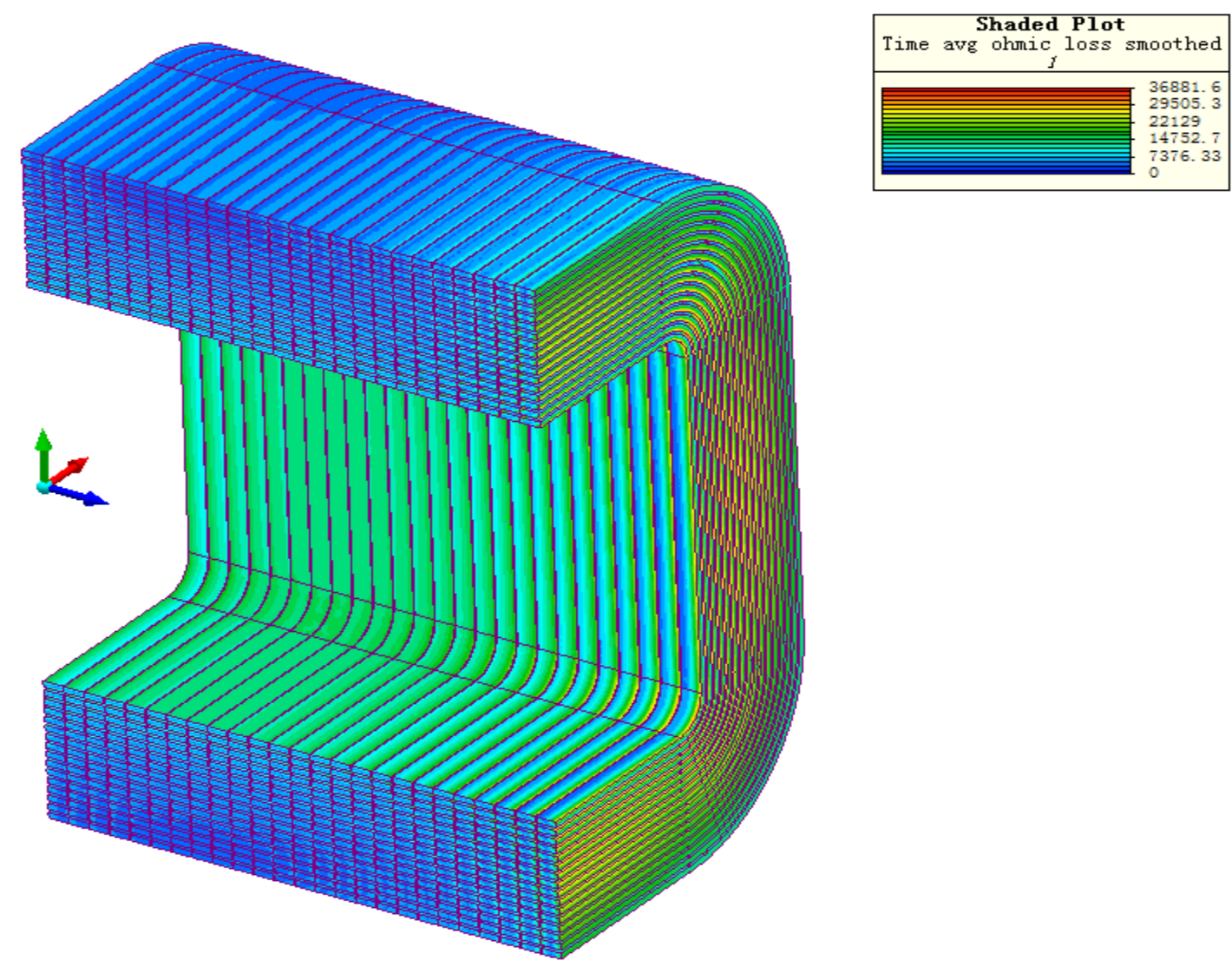

Figure 7. 3-D FE model for coil's eddy current analysis (by MagNet). 
Table 2. Loss in exciting coil under harmonics excitation.

\begin{tabular}{|c|c|c|c|c|c|c|}
\hline Currents $(\mathrm{A}, \mathrm{rms})$ & 5.0 & & 7.0 & & 9.0 & \\
\hline $\operatorname{Loss}(W)$ & Meas. & Calc. & Meas. & Calc. & Meas. & Calc. \\
\hline$P_{\sin }$ & 16.67 & 16.76 & 32.67 & 32.84 & 53.97 & 54.29 \\
\hline$P_{\text {harmonic }}$ & 18.38 & 19.52 & 36.04 & 38.28 & 59.60 & 63.28 \\
\hline
\end{tabular}

The measured and calculated loss results in the air-core exciting coils of model $\mathrm{P} 21^{0}-\mathrm{B}^{+}$, under the sinusoidal or the specified multi-harmonic excitations (as defined in (4)), are in practically good agreement. See Table 2.

The losses generated in the magnetic plate under the specified multi-harmonic excitations, as defined in (4), are obtained using different methods, i.e., the indirect determination based on the measured total loss and the calculated coil's loss (i.e., Approach III), and the numerical computation method. Both are also in practically good agreement. See Table 3.

Table 3. Loss in magnetic plate under harmonics excitation.

\begin{tabular}{|c|c|c|c|c|}
\hline $\begin{array}{l}\text { Currents } \\
(\mathrm{A}, \mathrm{rms})\end{array}$ & 5.0 & & 9.0 & \\
\hline \multirow[t]{2}{*}{$\begin{array}{l}P_{\text {harmonics }} \\
\text { (W) }\end{array}$} & $\begin{array}{l}\text { Indirectly } \\
\text { determined by } \\
\text { Approach III }\end{array}$ & & $\begin{array}{l}\text { Indirectly } \\
\text { determined by } \\
\text { Approach III }\end{array}$ & Calc. \\
\hline & 11.07 & 9.60 & 34.83 & 33.12 \\
\hline
\end{tabular}

\subsubsection{Non-Uniformity of Magnetic field and Loss}

According to the numerical computation results, the distributions of both the magnetic flux densities and magnetic loss in the cross-section of the ring specimens are not uniform due to skin effect. See Fig.8.

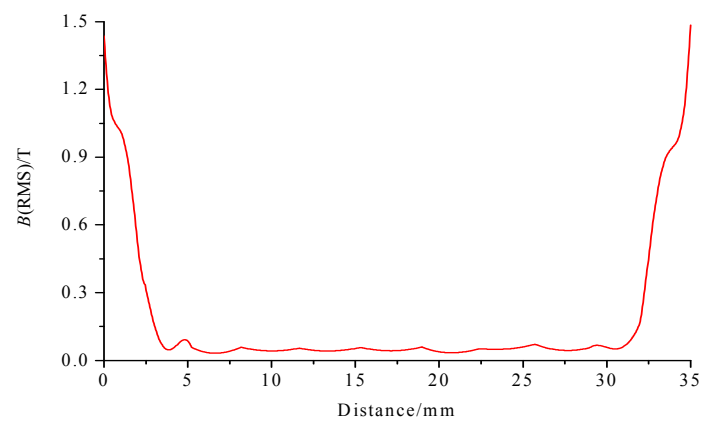

Figure 8. Distribution of magnetic flux densities (Q235B, RS1).

The heterogeneity, $\alpha$, of the magnetic flux results inside the ring specimens can be expressed by the following relation (5), i.e., the standard deviation of the magnetic flux densities divided by the average flux density over the cross-section of the ring specimen,

$$
\alpha=\frac{1}{B_{a}} \sqrt{\frac{\sum_{i=1}^{n}\left(B_{i}-B_{a}\right)^{2}}{n-1}}
$$

where, $B_{a}$ is the average value over all the magnetic flux densities (rms) over the cross section of the specimen; $B_{i}$ is the magnetic flux density (rms) at $i^{\text {th }}$ element; $\mathrm{n}$ is the total number of elements.
According to (5), the heterogeneity of the magnetic flux densities for the ring specimens RS1 under the multi-harmonic excitation (including $3^{\text {rd }}, 5^{\text {th }}$ and $7^{\text {th }}$ harmonics) is around 0.48 , and further examination shows that it is dependent on the average flux density and the width of the ring specimen. This fact should be considered in the accurate modeling and computation of the magnetic loss in the solid plate.

\section{Magnetic Loss in Laminated Component under Hybrid Excitation}

Smoothing reactors connected in series in HVDC systems are used to reduce the AC component and the transient over currents. In a magnetically-shielded smoothing reactor, there is a magnetic shielding frame outside the air-core winding to control the magnetic flux. The leakage flux enters the laminated frame from the air-core winding. This is essentially different from the usual case of a core-type power transformer.

In the stray-field loss evaluation in smoothing reactors, the measurement of the GO silicon steel material property under DC-bias with multiple harmonics, the multi-scale 3-D transient electromagnetic field analysis, due to the huge overall size of the structure and the very thin penetration depth in the magnetic parts, or the large laminated frame, are quite challenging. Nevertheless, the modeling and the prediction of the stray-field losses have become increasingly important.

This section investigates efficient numerical and reliable experimental approaches to be utilized in predicting the stray-field losses in smoothing reactors, including loss determination based on a 3-D transient field analysis, the measurement of the working magnetic properties under DC-bias and multi-harmonics, and validation based on a well-established smoothing reactor model and experimental system with DC-AC hybrid supply [17].

\subsection{Loss Calculation Inside the Shielding Frame}

In the smoothing reactor, the air-core exciting current $i(t)$ contains a heavy DC and multiple AC harmonics, as shown in (6),

$$
I=I_{D C}+\sum_{k} I_{m k} \cdot \cos \left(k \omega t+\phi_{k}\right)
$$

Where $I_{D C}$ is the DC component of the total exciting current, $\omega$, the fundamental angular frequency, and $I_{m k}$ and $\phi_{k}$, the amplitude and phase angle of the $k^{\text {th }}$ harmonic current, respectively.

Fig.9 shows the waveform of the DC-AC hybrid exciting currents, as an example, DC: $25 \mathrm{~A}$, and AC: $9 \mathrm{~A}$ (rms, the rated frequency: $50 \mathrm{~Hz}$, including $3^{\text {rd }}, 5^{\text {th }}$ and ${ }^{7 \text { th }}$ harmonics). 


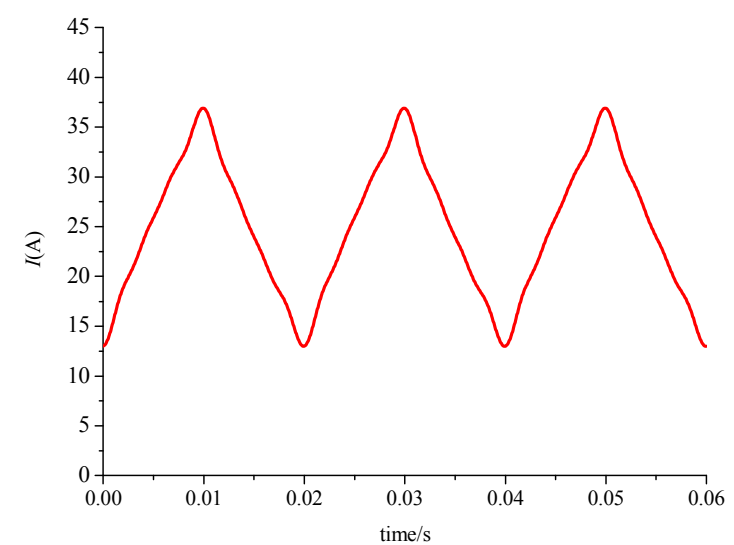

Figure 9. Hybrid exciting currents (DC: 25A; AC:9A,rated frequency: $50 \mathrm{~Hz}$, rms, including 3 harmonics).

The total magnetic loss of the laminated frame, $P_{\text {frame }}$, is calculated based on the 3-D transient field solution and the measured specific total magnetic loss over different zones, i.e., $P_{\text {limb }}\left(B_{m}, H_{D C}\right)$ in the middle section of the limb, $\Omega_{\text {limb }}$, and

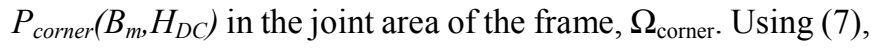
the magnetic loss in each element is calculated by interpolation, and then summed up for each zone,

$$
P_{\text {frame }}= \begin{cases}\sum_{\boldsymbol{e}} W_{u}^{(\boldsymbol{e})}\left(B_{m}^{(\boldsymbol{e})}, H_{D C}^{(\boldsymbol{e})}\right) \cdot V^{(\boldsymbol{e})} & \text { in } \Omega_{\text {limb }} \\ \sum_{\boldsymbol{e}} W_{n u}^{(e)}\left(B_{m}^{(\boldsymbol{e})}, H_{D C}^{(\boldsymbol{e})}\right) \cdot V^{(\boldsymbol{e})} & \text { in } \Omega_{\text {corner }}\end{cases}
$$

where $V^{(e)}, B_{m}^{(e)}$, the volume and magnetic flux density of the element $e$, respectively.

The MagNet-based scripts [13] are designed and used to compute the total loss in laminated frame.

\subsection{Measurement of Working Magnetic Properties under Hybrid Excitation}

In order to investigate the non-uniform specific total losses inside the shielding frame of a smoothing reactor under hybrid excitation, an efficient measurement method by means of two laminated core frames has been developed $[13,18]$. The two core frames, referred to as $\mathrm{CF} 1$ and $\mathrm{CF} 2$, are made of the same GO silicon steel, using the same joint type (multi-step lap), with the same lamination thickness and width, but different length; in fact, CF 2 is a scaled-down version of CF 1. The two core frames have different active volume(or mass), $m_{a 1}$ and $m_{a 2}$, respectively. See Fig. 10 .

Two assumptions are made: 1) the magnetic field and loss distribution over the corner regions of both CF 1 and CF 2 are identical, despite the difference in the length of the limbs in the two frames; 2) the magnetic field and loss are uniform over the middle section of each limb.

In order to realize the two assumptions, it is important to keep the identity of the magnetic flux density and the magnetic field intensity by DC inside both CF 1 and CF 2 under DC-AC hybrid excitations.

The specific magnetization loss, over the indicated uniform zones of the core limbs, can be determined from the difference between the absolute power losses $P_{\text {frame } 1}$ and $P_{\text {frame } 2}$, obtained from CF 1 and CF 2, respectively, and the corresponding total mass of the uniform zone, $\left(m_{a 1}-m_{a 2}\right)$, implying that the frame corners have no effect on $W_{u}$ as expressed by (8),

$$
W_{u}=\frac{P_{f r a m e 1}-P_{f r a m e 2}}{m_{a 1}-m_{a 2}}
$$

According to the assumptions made above, the total loss and volume (or mass) of the four corner regions of CF 1 are in fact equivalent to those of $\mathrm{CF} 2$. Therefore, the average specific total loss in the non-uniform region, $W_{n u}$, can be determined from the absolute total power loss, measured from CF $2\left(P_{\text {frame } 2}\right)$, and the corresponding active mass at the 4 corners of the total non-uniform regions of $\mathrm{CF}-1, m_{\text {corners }}$, as shown in (9),

$$
W_{n u}=\frac{P_{\text {frame2 }}}{m_{\text {corners }}}=\frac{P_{\text {frame2 }}}{m_{a 2}}
$$

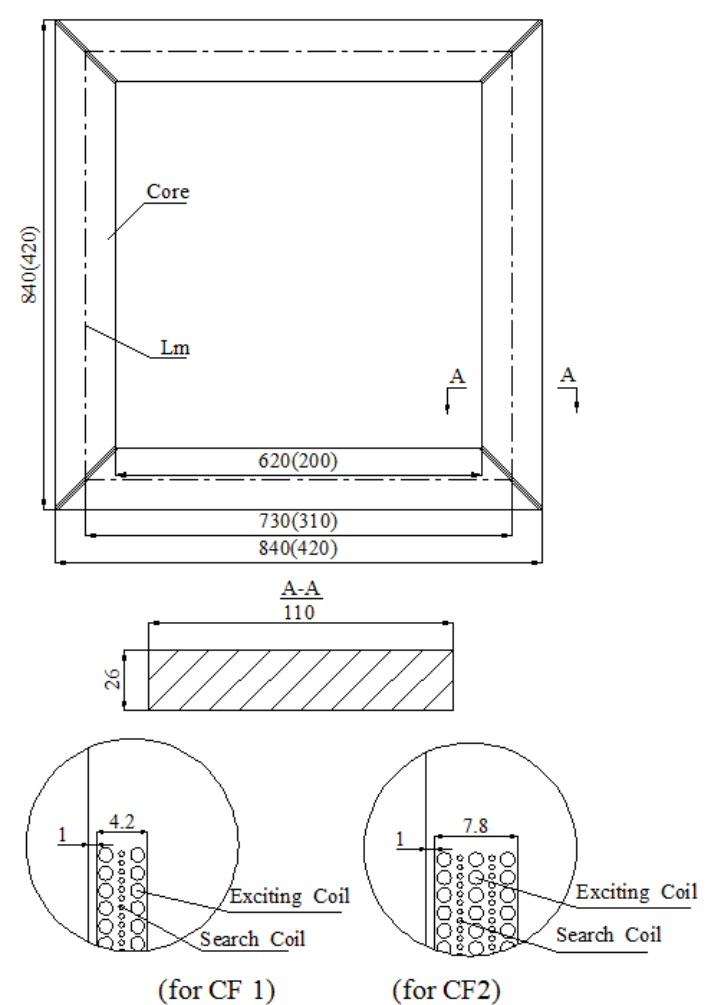

(a)

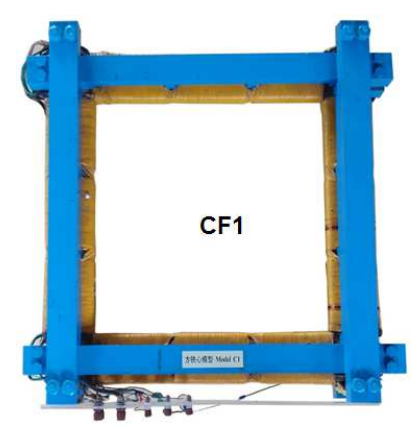




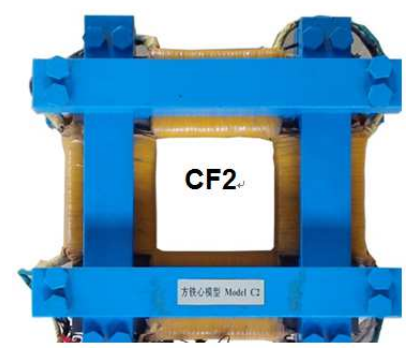

(b)

Figure 10. Measurement of magnetic property by using two core-frames, (a) dimensions of core-frames, and the numbers within parentheses () show the dimensions of CF 2; (b) tow core frames, $C F 1$ and $C F 2$ (photos).

The specific total loss curves measured at different DC-bias levels, described by $H_{d c}$, are shown in Fig.11. It can be observed that the specific total loss increases with the DC component of the total exciting currents, and the specific total losses at the corner zones (as expressed by $W_{n u}$ ) are greater than those (as expressed by $W_{u}$ ) at the middle of the frame's limb.

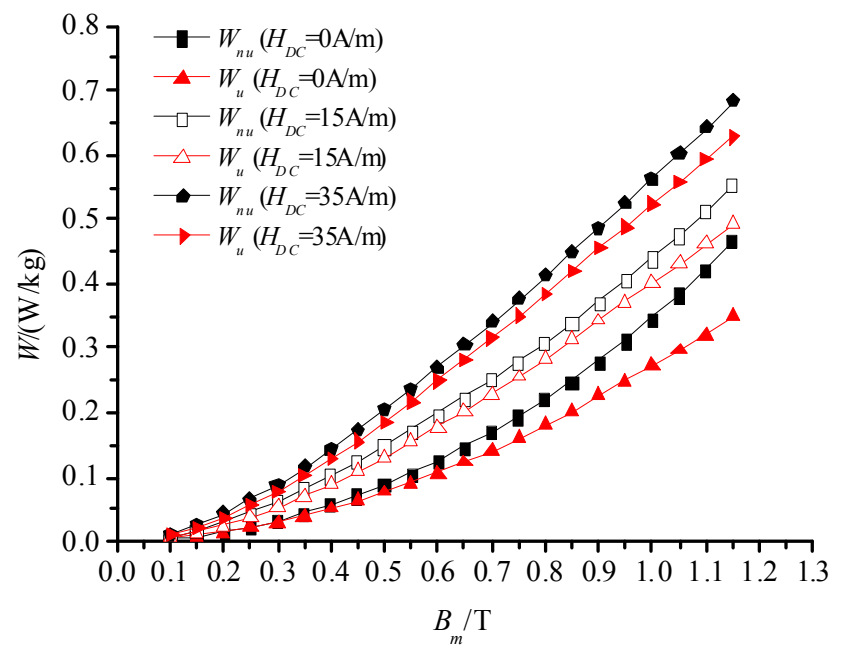

Figure 11. Specific total loss curves measured by two core frames (30Q140).

As mentioned above, the DC magnetization curve can be used in DC-bias transient filed analysis. Fig. 12 shows the DC magnetization curve of GO silicon steel 30Q140, provided by WISCO, Wuhan.

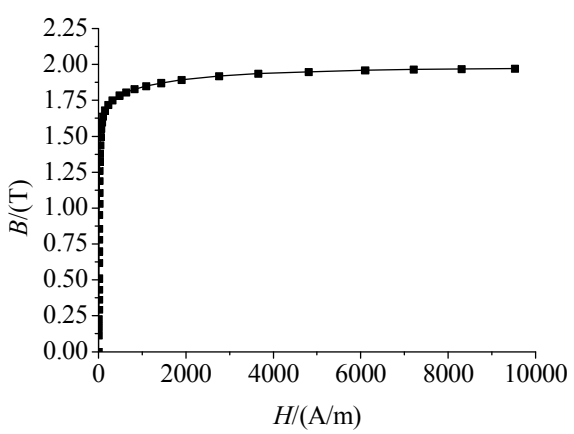

Figure 12. DC Magnetization curve (30Q140).

\subsection{Smoothing Reactor Model and Measurements}

\subsubsection{Smoothing Reactor Model}

The smoothing reactor model consists of an air-core elliptic exciting coil and the square laminated frame, CF 1, as used in the measurement of the magnetic property. See Fig.13. The main specification parameters of the exciting coil are: number of turns, 408; size of copper wire, $3 \times 9 \mathrm{~mm}$; conductivity of wire, $\sigma=5.7143 \times 10^{7} \mathrm{~S} / \mathrm{m}$.

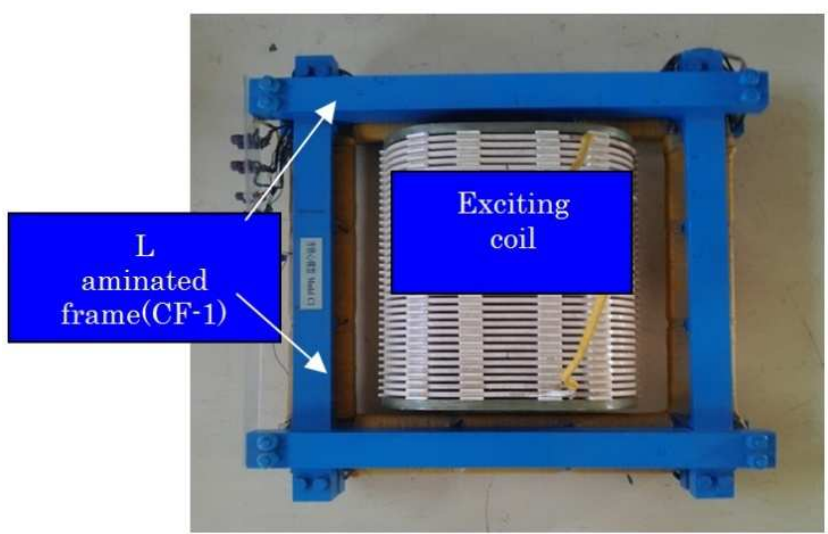

Figure 13. Smoothing reactor model.

\subsubsection{Loss Measurements under Hybrid Excitations}

The block diagram of the circuit used for measuring the stray-field loss, shown in Fig.14(a), includes the components: smoothing reactor model, multifunction generator (WF1974), precision power amplifier (4520/4520A), DC power supply (DH 400-37), NF Co., power analyzer (WT-3000, Yokogawa), Gauss/Teslameter (Model 7010, F.W.Bell), and multi-channel temperature recorder (TP700, Toprie Electronic). See Fig.14(b).

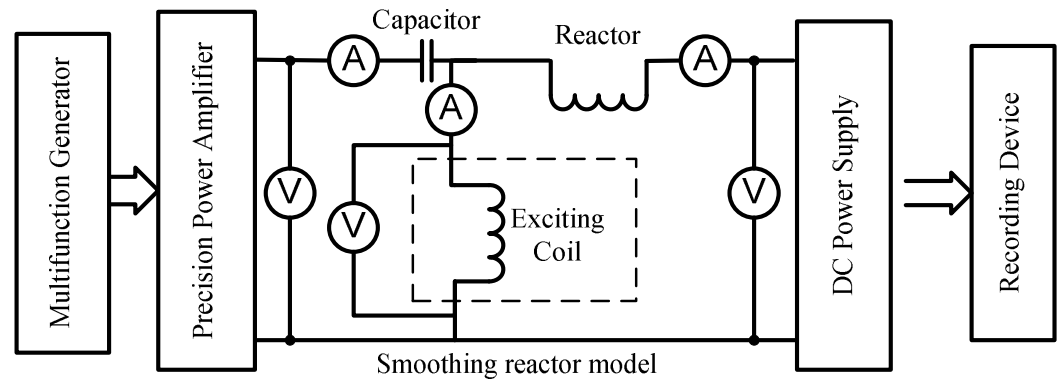

(a) 


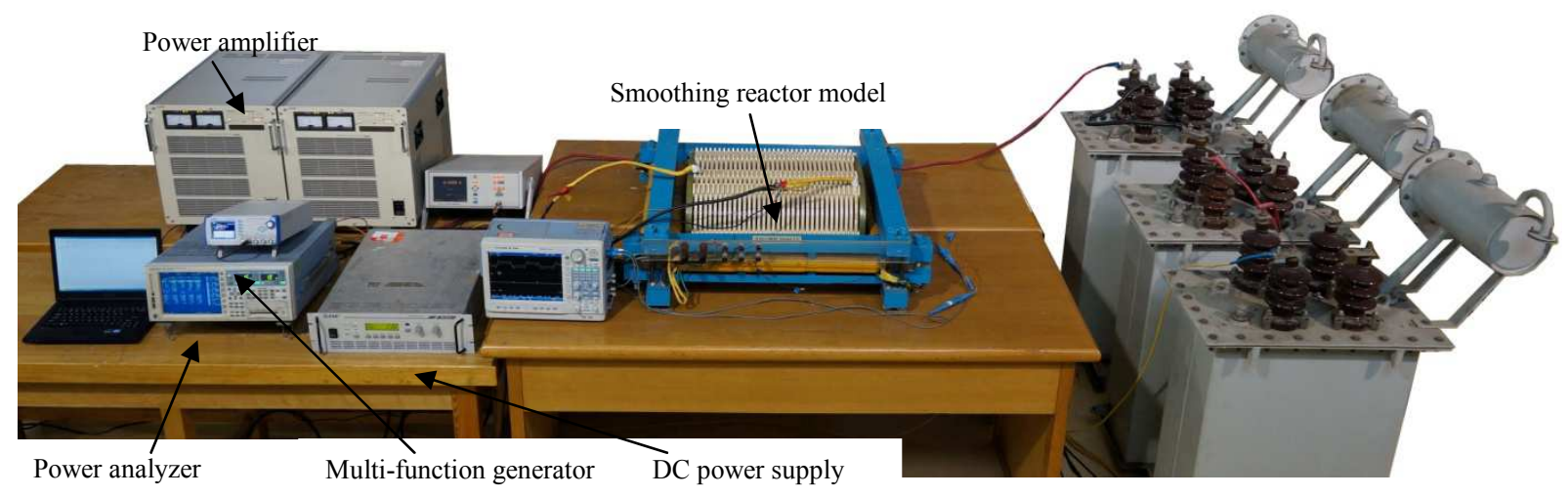

Figure 14. Measurement of stray-field loss in frame under hybrid excitations.(a) block diagram of the circuit; (b) experimental apparatus.

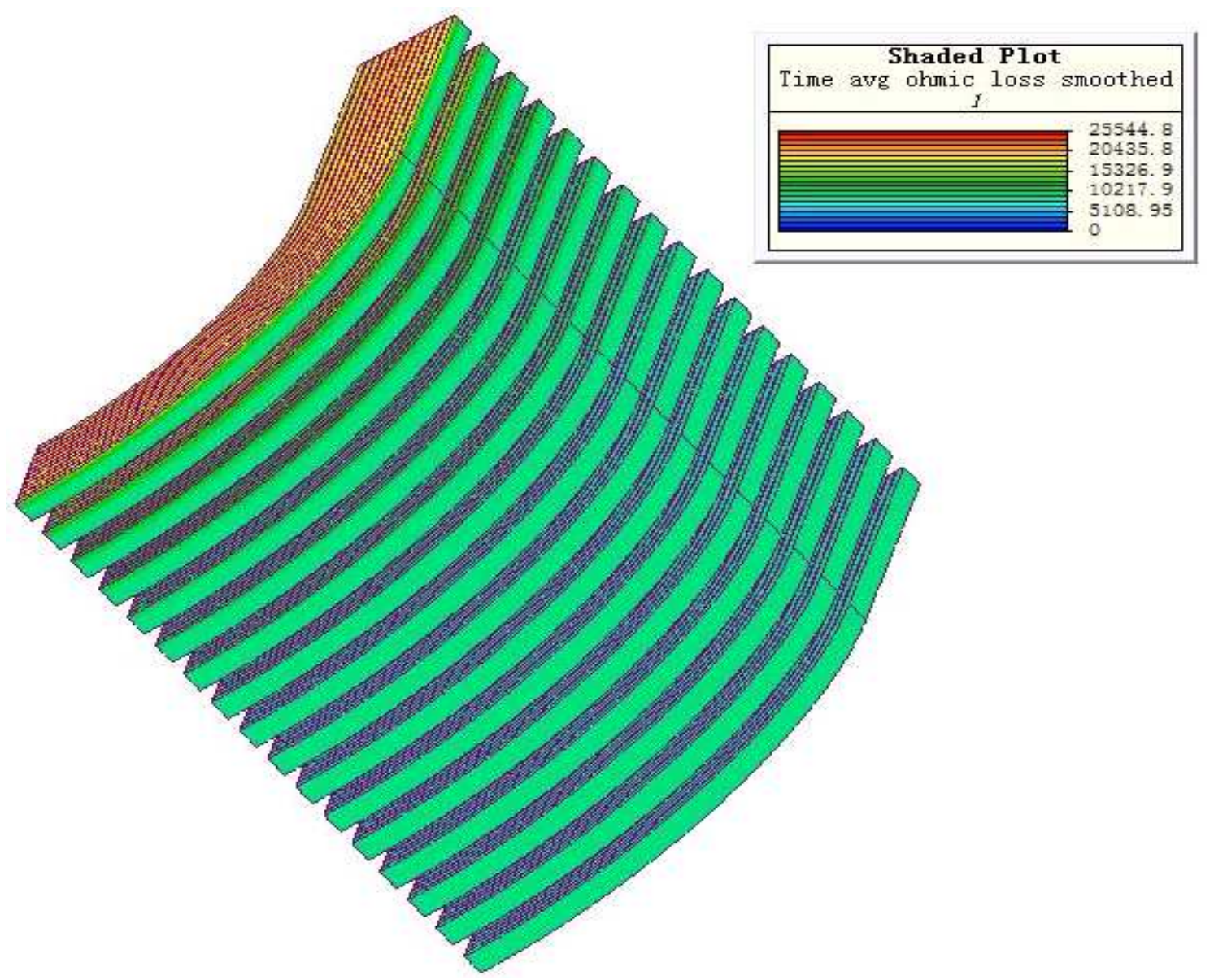

Figure 15. 3D FE model of air-core exciting coil.D

\subsection{Results and Discussions}

\subsubsection{Total Loss of the Air-Core Exciting Coil}

The total loss in the single air-core exciting coil, without the laminated frame, including both the eddy current and resistive loss, is measured using the power analyzer (WT3000, Yokogawa) and calculated using the FEA software (MagNet, Infolytica) under different excitations, including single and hybrid power supply. The solved finite element model, 1/8th of the whole coil, is shown in Fig.15. The corresponding coil's loss results are shown in Table 4.
The agreement between the calculated and measured loss results shows that the total loss of the air-core exciting coil can be accurately and numerically calculated under either single or hybrid excitations.

In order to further verify the coil's loss computation based on 3-D transient field solution, the leakage flux densities at the specified positions of the exciting coil, surrounded by the laminated frame and excited by DC-AC hybrid supply with 3 harmonics, i.e., DC:15A, AC: $9 \mathrm{~A}(\mathrm{rms})$, rated frequency $50 \mathrm{~Hz}$, with $3^{\text {rd }}, 5^{\text {th }}$ and $7^{\text {th }}$ harmonics, have been measured (by Gauss/Teslameter 7010, F.W.Bell, AC/DC can shift) and 
calculated (by MagNet, Infolytica), as shown in Fig.16. The agreement between the calculated and measured results demonstrates that the leakage flux linked with the exciting coil and then the induced eddy current loss can be accurately calculated under such complex excitation.

Table 4. Loss in air-core elliptic exciting coil.

\begin{tabular}{llll}
\hline Cases & Excitations & Calc.(W) & Meas. (W) \\
\hline I & $20.0 \mathrm{~A}(\mathrm{DC})$ & 119.03 & 118.57 \\
II & 20.0A(AC) & 127.06 & 126.99 \\
III & 20.0(DC), 6.9A(AC) & 126.88 & 125.29 \\
$*$ IV & 15A(DC) & 94.65 & 94.32 \\
& 9A(AC, multiple harmonics, rms) & & \\
$*$ V & 20A(DC) & 147.96 & 147.28 \\
& 9A(AC, multiple harmonics, rms) & & \\
$*$ VI & 25A(DC) & 217.22 & 215.02 \\
\hline
\end{tabular}

Note: *Cases IV-VI, under DC-AC hybrid excitation with multiple harmonics (AC: fundamental with $3^{\text {rd }}, 5^{\text {th }}$ and $7^{\text {th }}$ harmonic current components, rms).

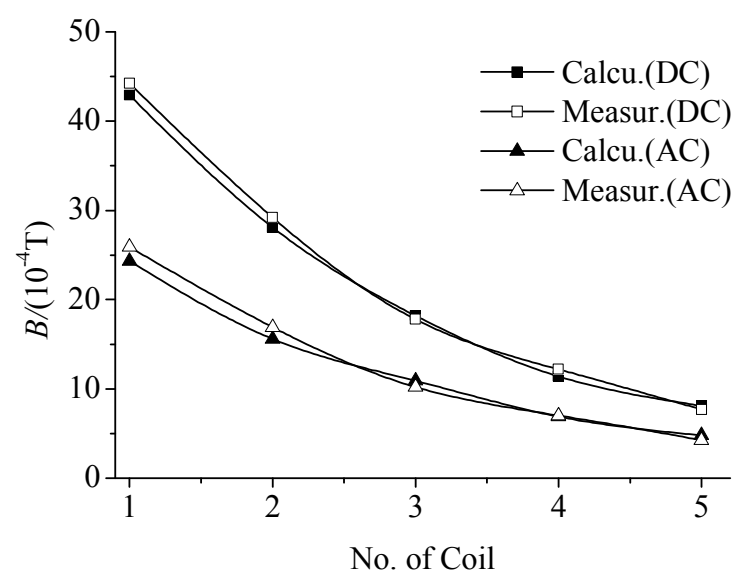

(a)

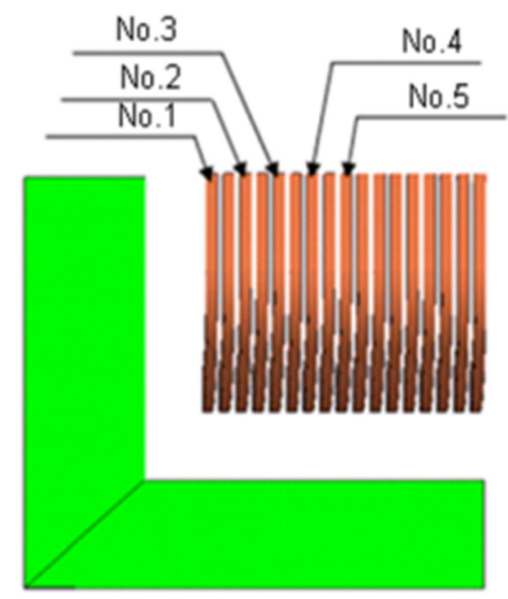

(b)

Figure 16. Leakage flux densities under hybrid excitations. (a) measured and calculated leakage flux densities; (b) specified measurement positions.

All the results presented herein provide a basic guarantee for the exciting coil's loss analysis. This is crucially important for indirect determination of component loss.

\subsubsection{Stray-Field Loss inside the Laminated Frame}

As described in Section II, the magnetic loss inside the laminated frame can be obtained using the two methods, i.e., using Approaches III and a numerical solver. The loss results involving 6 cases of DC-AC hybrid excitations are shown in Table 5, which are in practically good agreement.

Table 5. Loss in smoothing reactor model.

\begin{tabular}{lllllll}
\hline \multicolumn{2}{l}{ Exciting currents (A) } & \multicolumn{2}{l}{$\begin{array}{l}\text { Coil's loss } \\
(\mathbf{W})\end{array}$} & $\begin{array}{l}\text { Total loss } \\
(\mathbf{W})\end{array}$ & \multicolumn{2}{l}{ Loss in frame (W) } \\
\hline Cases & AC & DC & $\boldsymbol{P}_{\text {excitation }}$ & $\boldsymbol{P}_{\text {total }}$ & Calcu. & $\begin{array}{l}\text { Approach } \\
\text { III }\end{array}$ \\
\hline I & 15 & 15 & 142.94 & 143.82 & 0.77 & 0.88 \\
II & 15 & 18 & 178.29 & 179.24 & 0.87 & 0.95 \\
III & 20 & 15 & 200.19 & 201.64 & 1.33 & 1.45 \\
IV & 13 & 15 & 123.94 & 124.96 & 0.99 & 1.02 \\
*V & 9 & 20 & 147.10 & 148.02 & 0.85 & 0.92 \\
*VI & 13 & 20 & 177.50 & 178.63 & 1.07 & 1.13 \\
\hline
\end{tabular}

Notes: 1) Cases I-IV, DC+AC (only fundamental current without harmonics); 2)*Cases V-VI, under DC-AC hybrid excitation with multiple harmonics (AC: fundamental with $3^{\text {rd }}, 5^{\text {th }}$ and $7^{\text {th }}$ harmonic current components, rms).

The leakage flux densities normal to the laminations of the frame of smoothing reactor model have been measured and calculated at specified positions by the authors and reported elsewhere. The results suggest that the additional magnetic loss caused by such lower normal leakage flux can be neglected in the smoothing reactor model used in this paper.

\subsubsection{Further Research Works}

Until now the magnetic losses in both the solid and laminated components are evaluated using DC-AC hybrid supply at the lower excitation level.

Further examinations of the loss behavior inside both the solid and laminated components under much stronger hybrid excitations, and the effects of various factors on the total and local losses under complex excitations will be undertaken by the authors, including the magnetic anisotropy, the minor loops, the harmonic phase angles in the laminated components, and the multi-steel configuration in solid components.

\section{Conclusion}

The multi-scale 3-D transient modeling and analysis, the measurement of working magnetic properties of both material and component, the determination of magnetic loss under multi-harmonic and/or DC-basing excitation, and the experimental validation have been carried out, which can be briefly summarized as follows:

1) The magnetic loss inside both the solid and laminated components are calculated based on the 3-D transient field solution, using the developed scripts, and the specially measured specific total loss data obtained from the non-standard excitations.

2) The experimental and numerical method used for determining the exciting coil's loss under extreme excitations are realized. This provides the confident verification for finally determining the loss inside magnetic components. 
3) All the developed numerical and experimental approaches are validated, which can be effectively used in magnetic loss evaluation under extreme excitations.

4) The non-uniformity of the magnetic field and loss caused in solid plate configurations due to considerable skin effect, or in the laminated frame due to lamination-joints, must be taken into account for accurately determining the magnetic loss, under extreme excitation.

As a further research project, the magnetic property modeling under strong hybrid excitations, the detailed examinations of various effects on magnetic losses, and the efficient large-scale numerical modeling and simulation will be undertaken based on the upgraded models and enhanced experimental systems.

\section{Acknowledgement}

This project was supported in part by the National Natural Science Foundation of China under Grant 51107026, and by the State Grid Corporation of China under Grants sgri-wd-71-13-002, sgri-wd-71-14-002, and sgri-wd-71-14 -009 .

\section{References}

[1] F. Fiorillo and A. Novikov, "An improved approach to power losses in magnetic laminations under nonsinusoidal induction waveform," IEEE Trans. Magn., vol. 26, no.5, pp. 2904-2910, 1990.

[2] T. Sasaki, S. Saiki, and S. Takada, "Magnetic losses of electrical iron sheets under ac magnetization superimposed with higher harmonics", IEEE Trans. J. Magn. Japan, vol. 7, no. 1 , pp. $64-74,1992$.

[3] E. Barbisio, F. Fiorillo, and C. Ragusa: "Predicting loss in magnetic steels under arbitrary induction waveform and with minor hysteresis loops", IEEE Trans. Magn., vol. 40, no.4, pp.1810-1819, 2004.

[4] X. Zhao, J. Lu, L. Li, Z. Cheng, et al, "Analysis of the DC bias phenomenon by the harmonic balance finite element method," IEEE Trans. Power Delivery, vol.26. no.1, pp.475-485, 2011.

[5] Y.Yao, C.Koh, G.Ni, and D.Xie, "3-D nonlinear transient eddy current, calculation of online power transformer under DC bias," IEEE Trans. Magn., vol.41, no.5, pp.1840-1843, 2005.

[6] T. Moses and J. Leicht, "Prediction and Measurement of losses under PWM Magnetisation conditions in electrical steels with different silicon content," Journal of Applied Physics 97, 10R507 (2005).

[7] IEC TR 62383, First edition 2006-01, Determination of magnetic loss under magnetic polarization waveforms including higher harmonic components. Measurement, modelling and calculation methods.

[8] Z. Cheng, N. Takahashi, B. Forghani, G. Gilbert, J. Zhang, L. Liu, Y. Fan, X. Zhang, Y. Du, J. Wang, and C. Jiao, "Analysis and measurements of iron loss and flux inside silicon steel laminations," IEEE Trans. Magn.,vo.45, no.3, pp.1222-1225, 2009.

[9] Z. Cheng, N. Takahashi, B. Forghani, et al, "Effect of excitation patterns on both iron loss and flux in solid and laminated steel configurations," IEEE Trans. Magn., vol.46, no.8, pp.3185-3188, 2010.

[10] Y. Zhang, Z. Peng, D. Xie, and B. Bai, "Effect of different magnetization curves on simulation for transformer core loss under DC bias," Trans., China Electrotechnical Society, vo.29, no.5, pp.43-48, 2014.

[11] Z. Cheng, N. Takahashi, B. Forghani, Y. Du, Y. Fan, L. Liu, and H. Wang, "Effect of variation of B-H properties on both iron loss and flux in silicon steel lamination," IEEE Trans. Magn., vol.47, no.5, pp.1346-1349, 2011.

[12] Z. Cheng, N. Takahashi, B. Forghani, et al, "3-D finite element modeling and validation of power frequency multi-shielding effect," IEEE Trans. Magn., vol.48, no.2, pp.243-246, 2012.

[13] Z. Cheng, N. Takahashi, B. Forghani, et al, "Electromagnetic and Thermal Field Modeling and Application in Electrical Engineering," (in Chinese), ISBN 978-7-03-023561-9, Science Press, Beijing, 2009.

[14] IEC 60404-6, Magnetic materials- Part 6: Methods of measurement of the magnetic properties of magnetically soft metallic and powder materials at frequencies in the range $20 \mathrm{~Hz}$ to $200 \mathrm{kHz}$ by the use of ring specimens.

[15] TEAM Benchmark Problems (no.1-n0.34) [on line] available: www.compumag.org/team.

[16] Z. Cheng, N. Takahashi, B. Forghani, L. Liu, Y. Fan, T. Liu, Q.Hu, S. Gao, J. Zhang, and X. Wang, "Extended progress in TEAM Problem 21 family," COMPEL, 33, 1/2, pp.234-244, 2014.

[17] Z. Cheng, B. Forghani, D. Lowther, Y. Liu, Z. Zhao, T. Liu, Y. Fan, and G. Han, "Stray-field loss modeling under hybrid excitation in smoothing reactors", presented at 20th international Conference on the Computation of Electromagnetic Fields(Compumag), June 28-July 2, 2015, Montreal, Canada.

[18] Z. Cheng, N. Takahashi, B. Forghani, A. Moses, P. Anderson, Y. Fan, T. Liu, X. Wang, Z. Zhao, and L. Liu, "Modeling of magnetic properties of GO electrical steel based on Epstein combination and loss data weighted processing," IEEE Trans. Magn., vol.50, no.1, 6300209, 2014. 